\title{
Flexible microwave system to measure the electron number density and quantify the communications impact of electric thruster plasma plumes
}

\author{
B. E. Gilchrist and S. G. Ohler \\ Radiation Laboratory, University of Michigan, Ann Arbor, Michigan 48109-2144 \\ A. D. Gallimore \\ Plasma Physics and Electric Propulsion Laboratory, University of Michigan, Ann Arbor, Michigan 48105
}

(Received 22 May 1996; accepted for publication 11 November 1996)

\begin{abstract}
An advanced microwave interferometric system operating in the $\mathrm{Ku}(12-18 \mathrm{GHz})$ band has been implemented for use in very large vacuum chambers to determine the effects of electromagnetic wave propagation through a plasma plume created by a space electric propulsion thruster. This diagnostic tool is used to nonintrusively obtain the local electron number density as well as provide information necessary for understanding impact to communications and other spacecraft electromagnetic systems. The use of a nonintrusive electromagnetic measurement provides highly accurate line integrated density and avoids problems caused by intrusive measurement techniques. If the plasma is symmetrical, local plasma density can also be determined accurately using well known inversion techniques. A network analyzer acts as a transmitter and receiver while a two axis positioning system maps the amplitude and phase variation of a transmitted signal over one plane of the plasma plume. The utilization of a $6 \mathrm{~m} \times 9 \mathrm{~m}$ vacuum chamber effectively minimizes plasma boundary effects, but the longer cable path lengths have required a frequency conversion circuit to reduce power loss and phase uncertainty at high frequencies. Two studies are presented: the first is a measurement of the local electron density in the plume of a $1 \mathrm{~kW}$ arcjet and the second is a measurement of attenuation in the plume of a stationary plasma thruster. Both the arcjet and SPT emit a steady state conical unmagnetized plasma that is radially symmetric. The arcjet peak density is $10^{15}-10^{16} \mathrm{~m}^{-3}$ along centerline and the SPT peak density is $10^{16}-10^{17} \mathrm{~m}^{-3}$ along centerline. (C) 1997 American Institute of Physics. [S0034-6748(97)02602-6]
\end{abstract}

\section{INTRODUCTION}

Nonintrusive radio frequency diagnostics of plasma density and temperature are well recognized for their accuracy and speed of measurement in numerous fields such as fusion research, plasma processing, and studies of planetary ionospheres. ${ }^{1-4}$ Interest has also recently been shown in using microwave interferometric measurement techniques of plasma plumes generated by high energy electric propulsion (EP) thrusters. ${ }^{5-10}$ Modern EP thrusters are of special interest for next-generation satellite systems because of their high efficiencies and nearly optimal propellant exhaust velocity. ${ }^{11}$ Experimentally characterizing the interaction of a microwave signal with an EP thruster plume can provide a direct measure of line-integrated electron number density. Additionally, it is well known that plasma-microwave interactions can affect propagated electromagnetic signals in a number of ways including signal attenuation, added phase or amplitude noise, and refraction of signal energy. ${ }^{10}$ Therefore, a microwave experimental capability for EP thrusters in ground based chamber facilities or as part of flight experiments provides a method to nonintrusively map local plasma density as well as to characterize microwave signal degradation.

Microwave interferometry is a well established technique to measure line integrated electron number density. ${ }^{12-18}$ This technique is inherently nonintrusive and hence, avoids the issues of probe heating and local plasma perturbations of in situ techniques in dense energetic plasmas. Most present systems have a single, highly accurate measurement of line integrated density but must assume a spatial distribution in order to estimate local density from a single line integrated measurement. The results are sometimes used for calibration of Langmuir probes. ${ }^{19}$ A few systems provide spatial mapping in one plane of the plasma and use Abel inversion in that plane to find local density. It would be desirable to provide spatial mapping in two planes of the plasma for more complete characterization of the local plasma density. Additionally, present systems are limited when compensating for vacuum chamber effects such as chamber resonances or multipath since most use single frequencies and traditional phase detector circuits for the interferometric phase comparison. A partial solution to assessing and/or limiting chamber effects is through the use of a network analyzer as suggested by Birkner, ${ }^{5}$ although it apparently was not implemented. The second application mentioned above, quantifying the plasma impact to propagated electromagnetic signals, has received minimal ground based experimental measurements to date. ${ }^{6,8,10}$ A study has recently been completed which characterized the phase of a signal transmitted across an EP plasma plume at a single frequency for a number of geometries. ${ }^{6}$ It is desirable for a more complete assessment to also measure amplitude in addition to phase, and to do the measurements over a wide bandwidth.

Such a microwave measurement tool for EP generated plasmas should provide spatially resolved measurements in various orientations, quantify both magnitude and phase modification, and be able to address signal multipath and resonance effects in enclosed test facilities such as vacuum chambers. Additionally, for studies assessing impact to 
propagated electromagnetic systems, the diagnostic tool should cover a wide range of frequency bands and operate in sufficiently large vacuum facilities such that plasma boundary effects are minimized.

The measurement system described here addresses all of these considerations. We employ a microwave network analyzer (quadrature heterodyne receiver) which can accurately measure signal phase and magnitude with respect to a reference signal. It operates in the Ku frequency band to maintain adequate phase sensitivity (peak plasma frequency in the 1-3 GHz range). It utilizes a unique up-down frequency converter circuit to minimize measurement errors otherwise induced by operation in the Ku-band frequency range while still allowing broadband frequency operation required for communications impact studies. Because of a unique timegating feature of the network analyzer, it is also possible to compensate for signal multipath and resonances inside the vacuum chamber. ${ }^{20}$ Finally, a highly accurate probe positioning system allows detailed mapping through the plume.

The two plasma thrusters under test are the arcjet and the stationary plasma thruster (SPT) which produce conical plasma plumes where the SPT also has a beamlike nature close to the source (less than $1 \mathrm{~m}$ ). The arcjet produces a peak local electron number density between $10^{15}-10^{16} \mathrm{~m}^{-3}$ along the centerline which decreases by approximately half $45^{\circ}$ off centerline. ${ }^{15}$ The arcjet has electron and ion temperatures of $0.3 \mathrm{eV} .^{15}$ The SPT produces peak local electron number density between $10^{16}-10^{17} \mathrm{~m}^{-3}$ where the density is $10 \%$ of the peak value $45^{\circ}$ from centerline. The electron temperature is $3 \mathrm{eV}$ and the ion temperature is $0.1 \mathrm{eV}$. Due to the higher density and density gradients in the SPT plume, the effect on microwave signals is higher than with the arcjet for both phase and amplitude. The SPT effect on amplitude is primarily attributed to refraction of the microwave signal due to density gradients. ${ }^{10}$

Section II describes our measurement system in detail. Section III characterizes system performance. Section IV presents two measurements to demonstrate its use: phase and amplitude. Finally, Sec. V reviews the instrument's capabilities and comments on the flexibility and benefits of this microwave system.

\section{SYSTEM DESCRIPTION}

The Ku-band (12-18 GHz) microwave measurement system is composed of five primary components: the positioning system, support structure, the antennas, the frequency up-down conversion circuit, and the network analyzer. The network analyzer is essentially a highly sensitive heterodyne quadrature receiver. All of the components in Fig. 1 are placed in a vacuum chamber except the network analyzer. In the chamber, the positioning system moves the support structure. The support structure holds the antennas, connecting coaxial cable and conversion circuit. The conversion circuit is connected to the network analyzer through $15 \mathrm{~m}$ of flexible coaxial cable.

The vacuum chamber which is stainless steel and 9-mlong by 6-m-diam is located at the Plasmadynamics and Electric Propulsion Laboratory (PEPL) at the University of Michigan. The vacuum facility is supported by six $0.81-\mathrm{m}-$

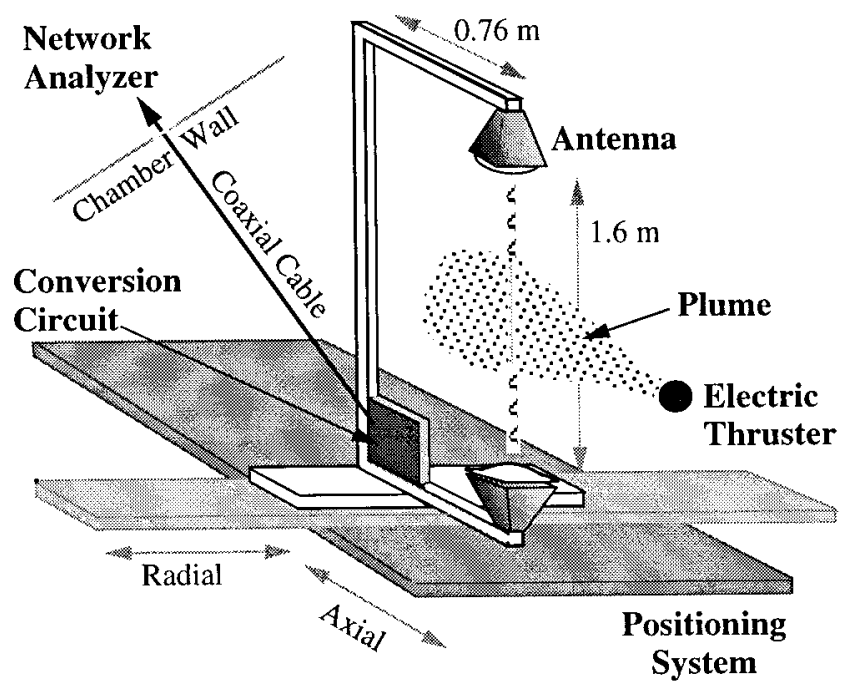

FIG. 1. Schematic of microwave measurement system.

diam diffusion pumps each rated at $32000 \mathrm{l} / \mathrm{s}$ (with watercooled cold traps), backed by two $2000 \mathrm{cfm}$ blowers, and four $400 \mathrm{cfm}$ mechanical pumps. The experimental facilities are described in more detail in Gallimore. ${ }^{21}$

A state-of-the-art positioning system provides the capability to spatially map plume parameters. The system is driven and monitored with a computer. The positioning system is mounted on a movable platform to allow for measurements to be made throughout the chamber. The positioning system contains two linear stages with $0.9 \mathrm{~m}$ of travel in the axial direction and $1.5 \mathrm{~m}$ of travel in the radial direction. The axial direction, shown in Fig. 1, is along the axis of the thruster. The radial axis indicates the direction orthogonal to the plane created by the thruster axis and the microwave transmission direction.

The steel support structure for the frequency conversion circuit, coaxial cable, and antennas is attached directly to the radial stage of the positioning table. The conversion circuit has been attached to the supports via a copper mounting plate which provides effective heat sinking. Semirigid coaxial cable attached to the steel supports connects the circuit to the horn antennas. The antennas are separated by $1.65 \mathrm{~m}$ and configured to transmit vertically through the plume. In addition to measurement components, graphfoil is used when necessary, in order to minimize sputtering of the support structure.

The horn antennas, which use dielectric lens correction, generate a narrow beam that transmits the signal through a narrow section of the plasma. The antennas are designed to minimize overall size while maintaining high gain. The antenna lens was designed so that its focal point was aligned with the phase center of the horn antenna, and then experimentally optimized to maximize power transmitted between the horns. The antennas have full angle half power beamwidths between $7^{\circ}$ and $8^{\circ}$ and approximately $25 \mathrm{~dB}$ gain for both the $E$-plane and the $H$-plane. Another antenna characteristic is that they exhibit negligible phase sensitivity to nearby dielectric or metallic scattering sites outside of the line-of-sight between the antenna structures. This fact indi- 


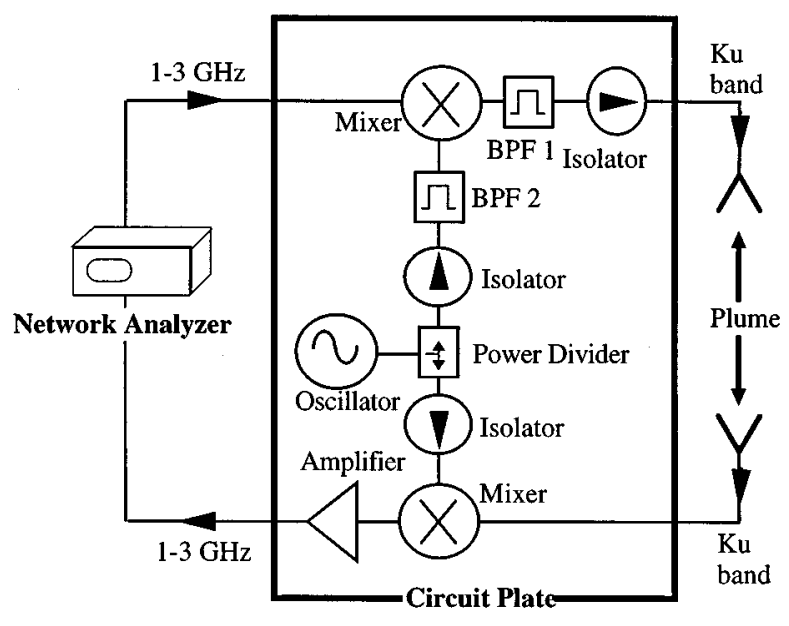

FIG. 2. Circuit diagram of circuit in microwave measurement system Fig. 1.

cates that the transmitted signal is essentially limited to a collimated beam $0.13 \mathrm{~m}$ in diameter (dimension of antenna). The antenna beam distribution will be addressed again in the next section in the discussion on calibration.

The frequency conversion circuit is utilized due to the long distance between the network analyzer outside of the chamber and the antenna system inside the chamber. The long distance produces unacceptable power loss and phase accuracy degradation when the Ku-band signal is transmitted directly through the $15 \mathrm{~m}$ of coaxial cable necessary to connect the network analyzer to the antennas.

The circuit in Fig. 2 receives a low frequency (1 to 3 $\mathrm{GHz}$ ) signal from the network analyzer, chooses the upper or lower sideband, transmits the signal to the antennas, receives the signal from the antennas, down converts to 1 to $3 \mathrm{GHz}$, and finally amplifies the power. The low frequency signal, 1 to $3 \mathrm{GHz}$, is converted to the $\mathrm{Ku}$ band via a mixer using a 15 $\mathrm{GHz}$ phase locked dielectric resonator oscillator. The oscillator supplies a reference signal to both the up and down mixing sides of the circuit thereby minimizing oscillator frequency drift and phase noise effects. The signal is guided by the power divider, isolators, and band pass filter number 2 . The isolators and band pass filter number 2 limit the effects of low-frequency signal leakage and reflection. The signal from the mixer includes both upper and lower sidebands either of which is available for use. The desired sideband is chosen by band pass filter number 1 . The upper sideband (16 to $18 \mathrm{GHz}$ ) is available with the present configuration. The circuit transmits less than $0.1 \mathrm{~mW}$ through the plume to the receive antenna. The received signal is then downconverted, amplified, and transmitted via coaxial cable back to the network analyzer.

This measurement system utilizes the capabilities of a network analyzer as a stable microwave source and highly sensitive heterodyne quadrature receiver in order to obtain postprocessing interferometric phase information. The network analyzer and the local oscillator are independently phase locked providing a stable signal both in phase and amplitude. The independence of the phased locked oscillators is not a problem since the phase of the conversion circuit is both inserted and removed from the signal during up and down conversion. The up-down frequency conversion scheme also allows the use of a much less costly lower frequency network analyzer. Additionally, network analyzers can isolate the test signal through the use of standard time gating techniques when the frequency is swept over a sufficiently wide bandwidth. ${ }^{20}$

\section{SYSTEM CHARACTERIZATION}

General base line characteristics provide information necessary for proper interpretation of measurements from the microwave system. Two measurable quantities, amplitude and phase, are characterized in terms of random noise as well as signal drift. A calibration function was developed to characterize the antenna propagation distribution through the use of a well known dielectric sample.

The amplitude and phase noise result primarily from slight variation in the microwave signal from the network analyzer and vibration of the support structure causing variation in transmitter-receiver alignment. The noise level in the network analyzer is specified by the ratio of the return signal to the transmitted power level. The power ratio is -20 to $-25 \mathrm{~dB}$ indicating network analyzer noise to be $\pm 0.5^{\circ}$ and $\pm 0.2 \mathrm{~dB}$. The received test signal at the analyzer is 20 to 25 $\mathrm{dB}$ below the transmitted signal from the analyzer resulting in an amplitude and phase of $\pm 0.2 \mathrm{~dB}$ and $\pm 0.5^{\circ}$, respectively. Mechanical vibration was found to introduce an additional $\pm 1.0^{\circ}$ resulting in final system level noise performance of $\pm 0.2 \mathrm{~dB}$ and $\pm 1.5^{\circ}$.

The drift in the signal is primarily caused by temperature changes in the components of the system; local oscillator, amplifier, mixer, and coaxial cable. The temperature of the circuit plate has been monitored in order to establish temperature induced drifts over time. In general, the circuit plate temperature increases $30{ }^{\circ} \mathrm{C}$ from ambient room temperature over the course of an initial $5 \mathrm{~h}$ period resulting in signal drift. The total steady state drift was found to be less than $0.08 \mathrm{deg}$ min and $0.06 \mathrm{~dB} / \mathrm{min}$ which is corrected in postprocessing.

An antenna calibration function was found to remove effects of finite antenna size. This is done by first calculating the theoretical phase shift through a foam cylinder and then comparing the theoretical results to the measured phase shift across the same cylinder. ${ }^{15,22}$ The theoretical calculation of phase shift for the cylinder ${ }^{2}$ is given by

$$
\Delta \phi_{\text {theory }}=\frac{180}{\pi} \frac{2 \pi}{\lambda}(n-1)\left(R^{2}-x^{2}\right),
$$

where $\Delta \phi_{\text {theory }}$ is the phase difference between a wave transmitted through free space and transmitted through the cylinder in degrees, $\lambda$ is the wavelength in meters, $n$ is the index of refraction, $R$ is the radius of the cylinder, and $x$ indicates the displacement of the transmission path from the center of the cylinder in meters. The index of refraction of 1.08 was estimated from the peak of the experimental results. This estimation does not affect the final conclusion concerning the effect on our measurement of local electron density. 
The calibration function $\mathrm{FN}$ relates the theoretical, $\Delta \phi_{\text {theory }}$, and experimental, $\Delta \phi_{\text {Exp }}$, phases mathematically by

$$
\begin{aligned}
& \Delta \phi_{\text {Exp }}(x)=\Delta \phi_{\text {Theory }}(x) * \mathrm{FN}(x), \\
& \mathrm{FN}=\frac{1}{\sqrt{2 \pi} \sigma} \exp \left[-\frac{1}{2}\left(\frac{x}{\sigma}\right)^{2}\right] .
\end{aligned}
$$

The distribution function is spatially convolved with the theoretical phase [Eq. (1)] to arrive at the measured results. The assumed Gaussian distribution function FN was optimized by varying the standard deviation $\sigma$. An optimal value of $\sigma$ equal to $0.024 \mathrm{~m}$ was found by minimizing the difference between the left and right sides of Eq. (2a). The fullwidth at half-maximum of the distribution function, which we take as a measure of system resolution indicates the resolution of the system is $2.36 \sigma$ or $0.057 \mathrm{~m}$. With this transfer function the effect of the finite size of the antenna beam are removed from the plasma measurements.

\section{APPLICATIONS}

This microwave measurement system has a number of applications in the characterization of plasmas. One significant application is to nonintrusively measure local electron number density using interferometric phase analysis followed by Abel inversion. In addition, characterizing the plasma effect on phase and amplitude provides information useful and necessary in communications impact studies. Here, we discuss measurements of signal attenuation to illustrate system capability. Additional measurements utilizing this system can be found in Refs. 10 and 15 .

\section{A. Microwave interferometry}

Local electron density is the result of an interferometric analysis of spatially resolved phase shift measurements which is followed by Abel inversion. ${ }^{9}$ Phase shift relates to the line integrated electron number density. Multiple measurements of the line integrated density provide information necessary to find local density using Abel inversion. The Abel analysis assumes a radially symmetric density distribution which is well founded in many plasmas such as electric propulsion plumes (in addition, any asymmetry exhibited by the thruster is not within the accuracy of this method). The symmetry of the plume is established through previous Langmuir probe measurements ${ }^{23}$ and through measurements of the line integrated density by the microwave interferometer on opposite sides of the plume. The Abel inversion formula, Eq. (3), is implemented by using a Gaussian curve fit for the phase distribution which results in a Gaussian density distribution. The Gaussian distribution is chosen due to its close approximation to the measurements:

$$
n_{e}(\rho)=-\frac{\lambda n_{c}}{\pi^{2}} \int_{\rho}^{R}\left(\frac{\partial \phi / \partial x}{\sqrt{x^{2}-\rho^{2}}}\right) d x .
$$

In the expression, $n_{e}$ is the local electron density, $\rho$ is the distance from the thruster axis, $n_{c}$ is the critical density for the operating frequency, and $\phi$ is the phase shift.

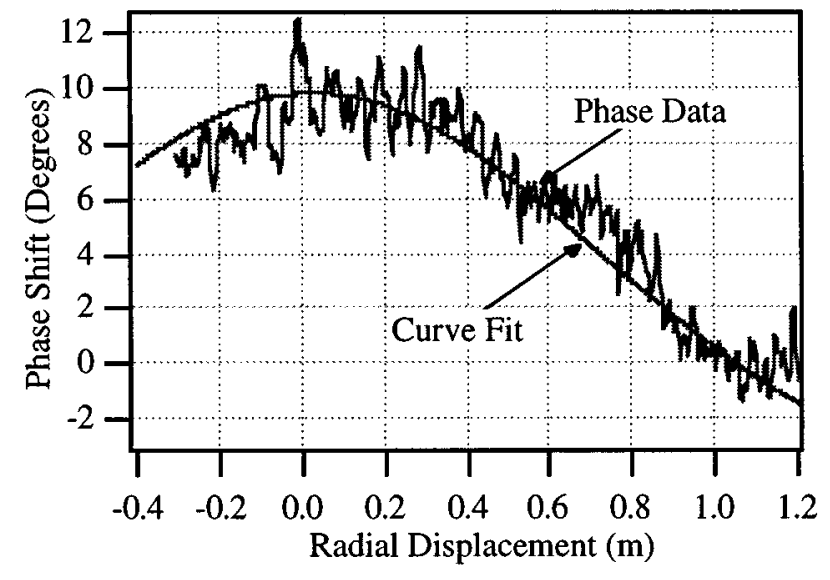

FIG. 3. Arcjet differential phase measurements compared to the Gaussian best fit.

Measurements of an arcjet local electron density have been completed using the microwave interferometer followed by Abel inversion and, for comparison, by a Langmuir probe in the plume of a $1 \mathrm{~kW}$ arcjet. ${ }^{9}$ The arcjet was run at $75 \mathrm{~V}$ and $8.8 \mathrm{~A}$ on 10 standard liters/minute $(16 \mathrm{mg} / \mathrm{s})$ of hydrogen at a chamber pressure of $4 \times 10^{-2} \mathrm{~Pa}\left(3 \times 10^{-4}\right.$ Torr). The phase data taken at $17 \mathrm{GHz}$ in a plane $0.66 \mathrm{~m}$ from the thruster exit plane and the Gaussian best fit to the data are plotted in Fig. 3 in order to demonstrate the close fit to the data. The truncated data in the acquired phase data is due to the microwave system offset from the positioning system center. The offset provided maximum possible displacement from the plume center. The phase measurements have been recorded at 600 points (4 data points per $\mathrm{cm}$ ). Next, the phase measurements are Abel inverted resulting in the local density distribution plotted in Fig. 4. The Langmuir probe is a $4.2 \times 10^{-3} \mathrm{~m}$ diameter by $5.1 \times 10^{-2} \mathrm{~m}$ long rhenium cylindrical probe. Langmuir probe measurements are analyzed through a standard Bohm sheath saturation current model since the probe radius to Debye length ratio is approximately twenty. ${ }^{21}$ Analysis of the Langmuir probe data

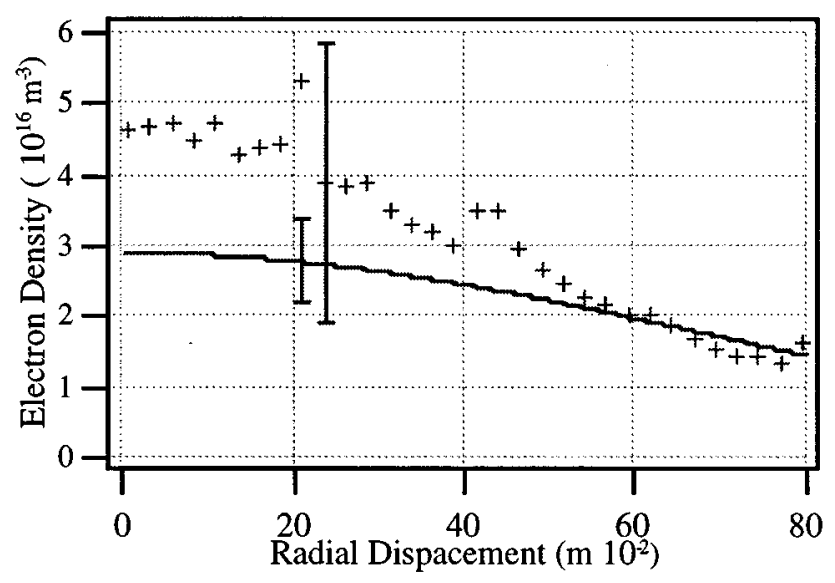

FIG. 4. Arcjet local electron density measurements from a microwave interferometer fitted to a Gaussian and a Langmuir probe at an axial distance of $0.66 \mathrm{~m}$. 


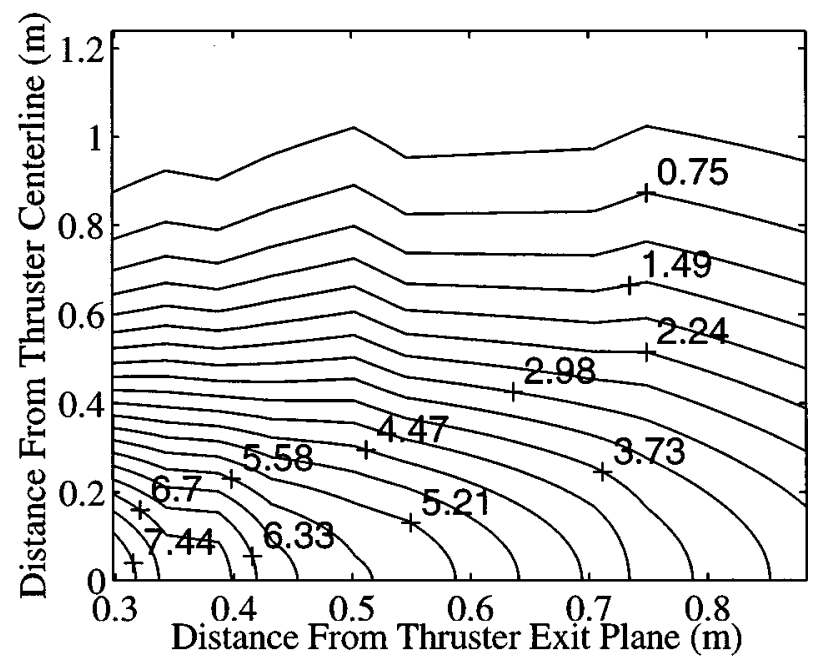

FIG. 5. Arcjet local electron density contours $\left(10^{15} \mathrm{~m}^{-3}\right)$.

results in an electron temperature of $0.1 \mathrm{eV}$, a plasma potential between 0.05 and $0.3 \mathrm{~V}$, and a saturation current between 5 and $25 \mathrm{~mA}$. The Langmuir probe density distribution is also plotted in Fig. 4 for comparison to the microwave interferometer and inversion local density results. The difference in the two data sets is within the accuracy of the two methods.

The accuracy of the two measurement methods relies on both theoretical errors and measurement errors. The microwave system is based on very accurate electromagnetic propagation through plasmas. The microwave measurement uncertainty is due to phase drift, phase noise, and positional uncertainty. The total uncertainty is estimated to be less than $20 \%$ of the peak local density through examining sources of error individually and summing the components. ${ }^{10}$ The Langmuir probe analysis relies on the accuracy of the Bohm sheath analysis. ${ }^{24}$ Experimental uncertainty is present in the probe flow alignment and signal noise in the voltage-current characteristics. Accuracy of the Langmuir probe was estimated at $50 \%$. $^{24}$

The two-dimensional mapping capability of the microwave interferometer is demonstrated through an electron density mapping for the arcjet operating with $15 \mathrm{mg} / \mathrm{s}$ of hydrogen and discharge parameters of $110 \mathrm{~V}$ and $10 \mathrm{~A}$. The contours have been developed through multiple radial sweeps at nine axial positions from 0.3 to $0.9 \mathrm{~m}$ as shown in Fig. 5.

\section{B. Attenuation study}

Signal attenuation due to beam refraction is determined through differential power measurements. The power level of the transmitted signal is measured with and without the plasma to obtain differential power. The basis of attenuation in the plume is that part of the antenna beam is refracted away from the receiver. The refraction occurs due to gradients in the electron density which indicate gradients in the dispersion function governing the propagation of electromagnetic waves. A possible secondary source of attenuation,

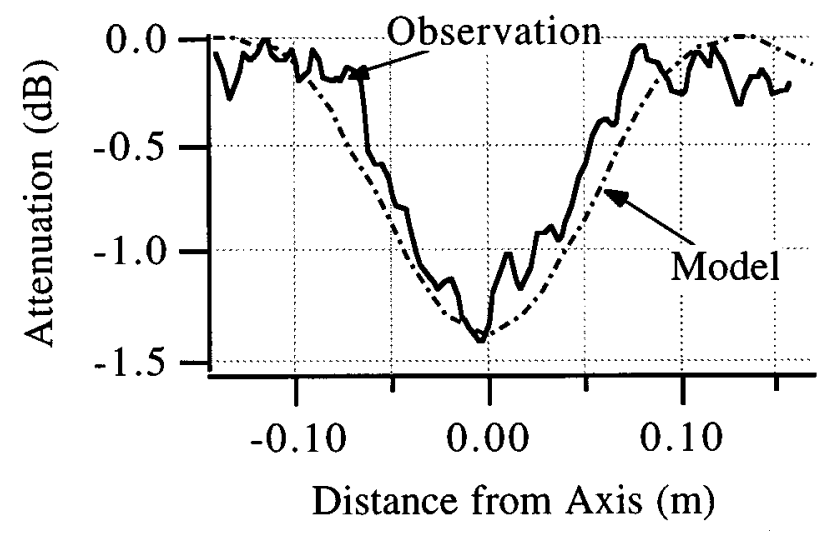

FIG. 6. Signal attenuation while propagating across a SPT plume at $0.15 \mathrm{~m}$ axially and $17 \mathrm{GHz}$ : measurements and ray trace modeling.

collisional damping, is minimal since the collision frequency which is less than $10 \mathrm{MHz}$ is much less than the signal frequency.

The attenuation measurements have been made on a SPT. ${ }^{9}$ The SPT was nominally operated at $300 \mathrm{~V}$ and $4.5 \mathrm{~A}$ using xenon with a flow rate of $5.8 \mathrm{mg} / \mathrm{s}$ of which $5 \%$ is directed to the cathode. During measurements, tank pressure was maintained below $7 \times 10^{-4} \mathrm{~Pa}\left(5 \times 10^{-5}\right.$ Torr $)$. Attenuation measurements in a plane $0.15 \mathrm{~m}$ from the thruster exit plane are plotted in Fig. 6 for a frequency of $17 \mathrm{GHz}$.

One use of the attenuation results can be to validate communications models of the thruster. With a local electron density distribution that could be obtained with the microwave interferometer, a ray tracing propagation code is able to model the plume effects on the transmitted signal. ${ }^{10}$ The modeling results, plotted in Fig. 6, agree closely with measurements. The attenuation measurements and modeling at $17 \mathrm{GHz}$ indicate minimal power loss for the microwave signal, however lower frequency transmission will experience much greater refractive effects and greater power loss.

\section{DISCUSSION}

A number of new aspects in the microwave characterization of plasmas have been demonstrated. A microwave interferometer has been implemented that can map the local electron density in two directions, and determine chamber effects such as resonances and multipath through the use of a network analyzer. The same system can be used for communications impact studies by measuring the amplitude and phase of a transmitted signal over a range of frequencies. The system is implemented in a large vacuum facility where chamber effects are minimized, and uses a frequency conversion circuit to eliminate problems with transmission of a high frequency signal through long coaxial cable lengths.

The system is demonstrated through characterization of a $1 \mathrm{~kW}$ arcjet and an SPT-100. The interferometric density measurements of the arcjet mapped the density in a plane orthogonal to the thruster axis and are compared to Langmuir probe measurements. A measurement of the SPT-100 which relates to communications issues is the characterization of the attenuation of a signal when transmitted across the 
plume. The attenuation measurement compares well with a ray tracing simulation using an electron density model of the plume.

\section{ACKNOWLEDGMENTS}

This research was funded in part by AFOSR Grant No. F49620-95-1-0331 (contract monitor: Dr. M. Birkhan). The authors would like to thank several students at PEPL including M. Domonokos, J. Foster, J. Haas, S. W. Kim, B. King, and C. Marrese whose help made the experiments happen. We would also like to thank M. Holladay of the Space Physics Laboratory for his work on coding the ray tracing model. Our appreciation also goes to Professor V. Liepa and Professor K. Sarabandi for their generous use of equipment. We wish to thank Dr. Frank Curran of NASA Lewis Research Center for the loan of the $1 \mathrm{~kW}$ arcjet and PPU. We wish to thank M. Day from Space Systems/Loral for the loan of the SPT-100.

${ }^{1}$ C. B. Heald and M. A. Wharton, Plasma Diagnostics With Microwaves (Wiley, New York, 1969).

${ }^{2}$ V. L. Ginzburg, The Propagation of Electromagnetic Waves in Plasmas (Pergamon, Oxford, 1970).

${ }^{3}$ J. Sheffield, Plasma Scattering of Electromagnetic Radiation (Academic, New York, 1975).

${ }^{4}$ H. Soltwick, Trans. Fus. Technol. 25, 304 (1994).

${ }^{5}$ G. A. Birkner, H. K. Hallock, and H. Ling, Rev. Sci. Instrum. 61, 2978 (1990).

${ }^{6}$ J. Dickens, Ph.D. thesis, Texas Tech University, 1995.
${ }^{7}$ J. Dickens, M. Kristiansen, and E. O'Hair, IEPC, Moscow, Russia, 1995, pp. $95-171$.

${ }^{8} \mathrm{~J}$. Dickens et al., 31st AIAA/ASME/SAE/ASEE Joint Propulsion Conference and Exhibit, San Diego, CA, 1995, AIAA 95-2929.

${ }^{9}$ S. Ohler, B. Gilchrist, and A. Gallimore, 30th AIAA/ASMA/SAE/ASEE Joint Propulsion Conference, Indianapolis, IN, 1994, AIAA 94-3297.

${ }^{10} \mathrm{~S}$. Ohler, B. Gilchrist, and A. Gallimore, 31st AIAA/ASME/SAE/ASEE Joint Propulsion Conference and Exhibit, San Diego, CA, July 1995, AIAA 95-2931.

${ }^{11}$ R. M. Myers and D. H. Manzella, 23rd International Electric Propulsion Conference, Seatle, WA, 1993, IEPC-93-096.

${ }^{12}$ D. Bora and P. K. Atrey, Rev. Sci. Instrum. 59, 2149 (1988).

${ }^{13} \mathrm{~S}$. W. Janson, 25th Plasmadynamics and Lasers Conference, Colorado Springs, CO, 1994, AIAA 94-2424.

${ }^{14}$ A. J. Kelly, AIAA J. 3, 372 (1965).

${ }^{15}$ S. G. Ohler, B. E. Gilchrist, and A. D. Gallimore, IEEE Trans. Plasma Sci. 23, 428 (1995).

${ }^{16}$ L. Overzet and M. Hopkins, J. Appl. Phys. 74, 4323 (1993).

${ }^{17}$ M. Sicha, J. Gajdusek, and S. Veprek, Br. J. Appl. Phys. 17, 15111 (1966).

${ }^{18}$ G. Neumann et al., Rev. Sci. Instrum. 64, 19 (1993).

${ }^{19}$ J. L. Cecchi et al., Lecture Notes for the Course "Production and diagnosis of process plasmas" (Princeton Scientific Consultants, Inc., Princeton, NJ, Nov. 1984).

${ }^{20}$ User's Guide: HP8753D Network Analyzer (Hewlett Packard, 1995), Chap. 6.

${ }^{21}$ A. Gallimore, M. Reichenbacher, and F. Guiczinski, AIAA J. Prop. Power 12, 105 (1996).

${ }^{22}$ S. W. Janson, 30th Joint Propulsion Conference, Indianapolis, IN, 1994, AIAA 94-2741.

${ }^{23}$ L. M. Carney and J. M. Sankovic, 25th Joint Propulsion Conference, Monterey, CA, 1989, AIAA-89-2723.

${ }^{24}$ D. L. Tilley, A. J. Kelly, and R. G. Jahn, 21st International Electric Propulsion Conference, Orlando, FL, 1990, AIAA 90-2667. 